\title{
Modeling KDP Bulk Damage Curves for Prediction of Large-Area Damage Performance
}

\author{
M. Runkel, R. Sharp III
}

This article was submitted to $31^{\text {st }}$ Boulder Damage Symposium: Annual Symposium on Optical Materials for High Power Lasers, Boulder, CO, October 4-7, 1999

U.S. Department of Energy

\section{December 16, 1999}

Lawrence

Livermore

National

Laboratory 


\section{DISCLAIMER}

This document was prepared as an account of work sponsored by an agency of the United States Government. Neither the United States Government nor the University of California nor any of their employees, makes any warranty, express or implied, or assumes any legal liability or responsibility for the accuracy, completeness, or usefulness of any information, apparatus, product, or process disclosed, or represents that its use would not infringe privately owned rights. Reference herein to any specific commercial product, process, or service by trade name, trademark, manufacturer, or otherwise, does not necessarily constitute or imply its endorsement, recommendation, or favoring by the United States Government or the University of California. The views and opinions of authors expressed herein do not necessarily state or reflect those of the United States Government or the University of California, and shall not be used for advertising or product endorsement purposes.

This is a preprint of a paper intended for publication in a journal or proceedings. Since changes may be made before publication, this preprint is made available with the understanding that it will not be cited or reproduced without the permission of the author.

This report has been reproduced

directly from the best available copy.

Available to DOE and DOE contractors from the

Office of Scientific and Technical Information

P.O. Box 62, Oak Ridge, TN 37831

Prices available from (423) 576-8401

http://apollo.osti.gov/bridge/

Available to the public from the

National Technical Information Service

U.S. Department of Commerce 5285 Port Royal Rd., Springfield, VA 22161

http://www.ntis.gov/

OR

Lawrence Livermore National Laboratory

Technical Information Department's Digital Library

http://www.llnl.gov/tid/Library.html 


\title{
Modeling KDP bulk damage curves for prediction of large-area damage performance
}

\author{
Mike Runkel and Richard Sharp III \\ L-250, Lawrence Livermore National Laboratory \\ 7000 East Avenue, Livermore CA, 94550
}

\begin{abstract}
Over the past two years extensive experimentation has been carried out to determine the nature of bulk damage in KDP. Automated damage testing with small beams has made it possible to rapidly investigate damage statistics and its connection to growth parameter variation. Over this time we have built up an encyclopedia of many damage curves but only relatively few samples have been tested with large beams. The scarcity of data makes it difficult to estimate how future crystals will perform on the NIF, and the campaign nature of large beam testing is not suitable for efficient testing of many samples with rapid turn-around. It is therefore desirable to have analytical tools in place that could make reliable predictions of large-beam performance based on small-beam damage probability measurements.
\end{abstract}

To that end, we discuss the application of exponential and power law damage evolution within the framework of Poisson statistics in this memo. We describe the results of fitting these models to various damage probability curves on KDP including the heavily investigated KDP214 samples.

We find that both models are capable of fitting the damage probability S-curves quite well but there are multiple parameter sets for each model that produce comparable $\chi^{2}$ values. In addition, the fit parameters from the exponential model do not agree well with the measured evolution from large-beam OSL experiments where pinpoint density was shown to evolve according to $n(F)=n_{0} \exp (b F)$. The largest discrepancy is in determination of the $b$ values. For the O'Connell formalism of the power law case developed here, we find that the best-fit powers have approximately the same magnitude as the Weibull exponent of Feit's formalism, but it is difficult to extract information about the defect concentration using the O'Connell approach. In addition, we found that the power law case provides slightly better $\chi^{2}$ values in roughly half of the cases.

We discuss these results in terms of fluence measurement precision and the observed fluctuation of damage density observed on OSL testing. We conclude that these two formalisms are not yet well-enough developed to provide reliable parameters for predicting large-scale damage performance of KDP. In outlining possible steps for refining the models, we also call for any future small-spot damage model to include the effects of laser conditioning.

Keywords: KDP, bulk damage, damage statistics, exponential evolution, curve fitting

\section{INTRODUCTION}

The statistical nature of laser damage plays a fundamental role in determining the survivability of large optics on the National Ignition Facility (NIF) laser. A firm understanding of the "extreme statistics" of laser damage is required to predict the amount and severity of damage expected on each type of optic in the laser. This has bearing not only on the single-shot performance of the laser, but also on the operational lifetime of the system optics. The damage groups are currently involved in an intensive effort to understand the statistical nature of laser damage and apply it to large optics in order to predict laser performance and optics lifetime on the NIF. The optics primarily under consideration are KD*P triplers and fused silica lenses. Isolated point-like absorbers appear to be the dominant source of damage in both cases although $\mathrm{KD}{ }^{*} \mathrm{P}$ exhibits primarily a bulk damage problem while fused silica damages mainly on the surfaces. To investigate the statistical nature of laser damage, the damage groups have constructed several automated damage test systems. These table-top, small beam systems $\left(\sim 1 \mathrm{~mm}, \mathrm{FW} @ 1 / \mathrm{e}^{2}\right)$ are capable of testing hundreds of sites to failure on each sample. This allows a more statistically valid picture of the damage performance to emerge than previously possible. As a result, the concept of "damage threshold" has given way to the concept of damage distributions. The current "standard" method of gauging performance is the cumulative failure probability curve ${ }^{1}$ or S-curve, which is a plot of failure probability versus fluence for a given beam size. This curve allows the large scale/area performance of an optic to be predicted using either reliability analysis methods ${ }^{2}$ 
or Weibull/Poisson (WP) statistics. Because the reliability analysis method has already been developed for KDP, we concentrate on the Weibull/Poisson statistical model in this memo. In what follows, we shall develop the basic Poisson statistical model, modify the model for damage evolution of KDP and long focus gaussian beam geometry, and describe the computer models used to fit theory to data. The results of data fitting will be discussed to determine the overall utility of the model in connecting small and large area test result and for predicting large area behavior from a sample's S-curve.

\subsection{Poisson statistical model and the Weibull (power law) formulation}

In a recent report ${ }^{3}$, Feit described the basic formalism of Poisson statistics applied to defect-driven damage. He then proceeded to develop it along the lines of the Weibull formalism ${ }^{4}$. The Poisson statistical approach is well established in the literature and variations of this approach has been developed previously ${ }^{5-13}$. However, in order to conceptualize what follows and establish notation, we repeat the basic derivation here.

Consider a surface area, $S$, which contains distribution of defects which may damage in $[F, F+d F] i . e . n(F) d F$, irradiated by fixed (top-hat) fluence, $F$. Let $P(S, F)$ be the probability to damage at $F$. The complementary probability to survive exposure to $F$ is $U(S, F)=1-P(S, F)$. The differential failure probability, $d P$, in the interval $[F, F+d F]$ is given by $-d U=n(F) S U(S, F) d F$. Integration of this equation yields the damage probability as a function of fluence:

$$
P(S, F)=1-\exp \left[-S \int_{0}^{F} n(F) d F\right]=1-e^{-S c(F)}=1-e^{-N}
$$

Note that the product of the area and defect concentration, $S c(F)=S \int_{0}^{F} n(F) d F$ is the total number of defects, $\mathrm{N}$, which damage up to $F$. The form of $P(S, F)$ depends explicitly on the functional form chosen or determined for $n(F)$ and whether $S$ depends on the fluence, as in the case of a gaussian beam. This is the basic functional form that describes defect driven damage. It can be put into Weibull form ${ }^{4}$ by choosing a power law dependence for $c(F)$, i.e. $c(F)=C F^{m}$ where $m$ is known as the Weibull exponent. Thus (1) becomes

$$
P=1-\exp \left(-S C F^{m}\right)
$$

where $\mathrm{C}$ is a constant. This expression can be manipulated to yield a linear form by applying laws of logarithms:

$$
\ln \left[\frac{1}{S} \ln \left(\frac{1}{1-P}\right)\right]=m \ln (F)+\ln C
$$

Here, $m$ is the slope of a straight line.

Interpretation of the data from a Weibull plot is not straightforward for small beam, gaussian laser systems. This is because the area above damage threshold depends on the peak intensity of the gaussian beam irradiating the surface. In the Weibull formalism this can be accounted for by evaluating $\mathrm{N}(\mathrm{area})$ :

$$
N=\int_{A} c(F) d A=C F_{0}{ }^{2} \int_{0}^{2 \pi} d \theta \int_{0}^{\infty} r e^{-m \frac{r^{2}}{\sigma^{2}}} d r=C F_{0}{ }^{m} \frac{\pi \sigma^{2}}{m}
$$

This defines the effective area of the gaussian beam as $A_{\text {eff }}=\pi \sigma^{2} / m$ for the gaussian beam $F(r)=F_{0} \exp \left(-r^{2} / \sigma^{2}\right)$ applied to a particular optic. This relation makes it clear that for high $\mathrm{m}$ optics (such as fused silica) large areas must be tested to reveal the true low fluence character of the failure curve.

This formalism is currently being used by Feit, Rubenchik and others to analyze the results of raster scanning fused silica at low fluences, where only a few damage events occur for many thousands of test sites ${ }^{5}$. For that problem, the Weibull formalism seems to be well suited as an analytical tool. The applicability of Weibull statistics to KDP damage was called into question, however, by the exponential evolution of pinpoint density as shown in Figure 1 below, which is the result of testing on LLNL's Optical Sciences Laser (OSL) ${ }^{14}$. 
As we show below, the damage behavior of KDP and DKDP can be described by exponential pinpoint generation as a function of fluence i.e. $n(F)=n_{0} \exp (b F)$. To our knowledge, this represents the first time a non-power law function, determined from actual measurements, has been used in this method. We would also like to point out that this behavior has been predicted by Duxbury et al. ${ }^{17}$ Previous efforts have postulated damage evolution according to a power law but do not dwell on its physical significance. In developing damage models for bulk damage in KDP, we shall apply the O'Connell formalism ${ }^{13}$.

\section{POISSON MODEL APPLIED TO KDP DAMAGE}

\subsection{Determination of damage evolution and $n(F)$ for KDP}

Recently, large beam damage experiments were performed on KDP using the Optical Sciences Laser (OSL) ${ }^{2,14}$. The tests were designed to examine the feasibility of on-line laser-conditioning and damage evolution of NIF THG crystals. Samples were exposed to a $7 \mathrm{~mm}$ diameter, $351 \mathrm{~nm}, 3 \mathrm{~ns}$, near top-hat beam to better match the statistics of the NIF beam compared to small-beam automated tests. The degree of conditioning was measured by exposing separate sites to increasing average fluence in $1 / 1,4 / 1,8 / 1$ and $12 / 1$ sequences to the same ending average fluence. Here N/1 refers to the number of shots taken on a given test site to step up to the final average fluence. Bulk scatter mapping revealed that significant conditioning was attained in 12/1 exposure sequences. It was also discovered that bulk damage evolution could be described by a simple exponential model relating the pinpoint density to local beam fluence: $n(F)=n_{0} \exp (b F)$. Figure 2 shows test data from the OSL campaign and the corresponding exponential fit for rapid-growth sample KDP214, which is one of the best rapid growth samples that LLNL has ever produced. Despite the general exponential behavior, the pinpoint density varies by up to two orders of magnitude for a given fluence. This variation only allows determination of $n_{0}$ and $b$ in an average sense.

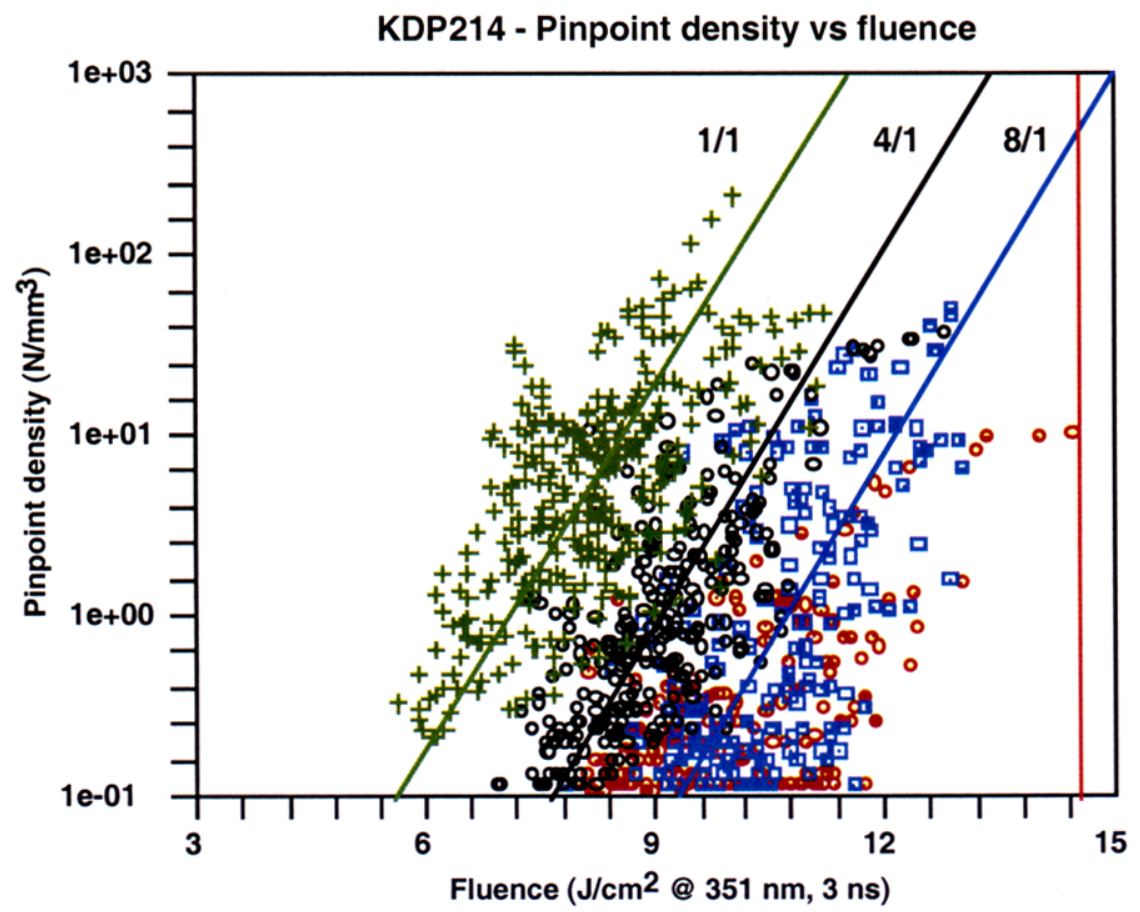

Figure 1. OSL damage evolution data for rapidly grown sample KDP214. The data show a reduction in damage density as well as an increase in threshold as a result of laser conditioning. The damage density (pinpints $/ \mathrm{mm}^{3}$ ) data were obtained by correlating measured (via microscope) densities with scatter voltage signal. This data was then correlated to the local beam fluence. Spatial resolution of beam fluence maps, microscopy and scatter maps were all $\sim 300 \mu \mathrm{m}$ [14].

To apply the Poisson formalism to small area KDP damage measurements, we follow O'Connell's notation and write equation (A9) from Appendix 1 in the form 


$$
P=1-\exp \left[-\int_{0}^{F_{0}} n^{\prime}\left(F^{\prime}\right) V\left(F^{\prime}\right) d F^{\prime}\right]
$$

Here $n^{\prime}(F)$ describes the change in damage density as a function of the fluence. $V(F)$ describes the evolution of the volume element in which damage will occur as beam fluence is increased. The integral is taken from zero up to the peak fluence in the beam.

To develop an explicit form for KDP, the derivative of the expression for exponential damage evolution is inserted into equation 5. To complete the model, we must also consider the form of the $V(F)$ term. The Zeus damage tests are performed under conditions where the focal waist of the beams is much greater than the sample thickness. Therefore, a cylinder of material is illuminated at each test site. If we introduce a local damage threshold fluence, $\mathrm{F}_{\mathrm{th}}$, which represents the welldefined, but unknown damaging fluence at the test site, then the radius of the irradiated cylinder where damage may occur (i.e. where $\mathrm{F} \geq \mathrm{F}_{\mathrm{th}}$ ) will increase with fluence as shown in Figure 2.

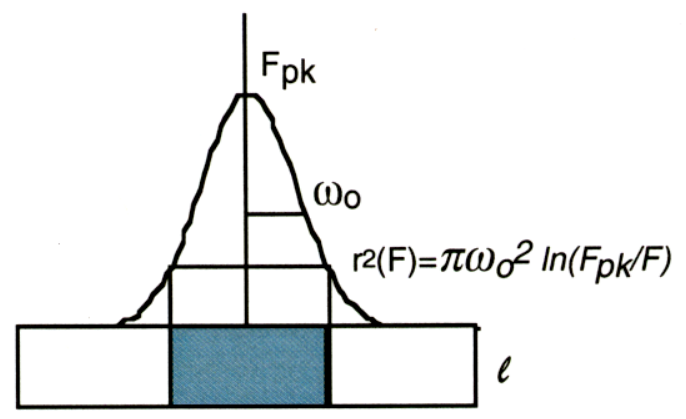

Figure 2. Geometry for irradiated volume at fluences above the local damage threshold, $\mathrm{F}_{\mathrm{th}}$.

Inverting the functional form of the gaussian spatial profile, $\mathrm{F}(\mathrm{r})=\mathrm{F}_{\mathrm{pk}} \exp \left(\mathrm{r}^{2} / \omega_{0}{ }^{2}\right),\left(\omega_{0}\right.$ denotes the distributions half-width at $1 / \mathrm{e}, 1$ is the sample thickness and $\mathrm{F}_{\mathrm{pk}}$ is the peak fluence in the beam) allows us to calculate $V(F)$.

$$
V(F)=\pi \omega_{0}^{2} l \cdot \ln \left(\frac{F_{p k}}{F}\right), \mathrm{F} \geq \mathrm{F}_{\mathrm{th}}
$$

The probability expression becomes

$$
P=1-\exp \left[-\pi \omega_{0}^{2} l \int_{F_{t h}}^{F_{p k}} n_{0} b \exp \left[b\left(F^{\prime}-F_{t h}\right)\right] \ln \left(\frac{F_{p k}}{F}\right) d F^{\prime}\right] \quad, \mathrm{F} \geq \mathrm{F}_{\mathrm{th}}
$$

Note that the introduction of $\mathrm{F}_{\mathrm{th}}$, which now represents the lowest failure fluence in the data set, requires modification of the evolution term. We now have an expression with three fitting parameters, $\mathrm{F}_{\mathrm{th}}, \mathrm{n}_{0}$ and $\mathrm{b}$ for evaluation of experimental data.

\subsection{Fitting the model to experimental data}

We took two approaches in fitting the exponential model to experimental data. The first was to evaluate the probability expression (7) using straightforward numerical integration on a spreadsheet, which allowed us to rapidly explore the behavior of the models over a wide parameter range. The second approach we developed consisted of a non-linear least squares fitting program based on the Levenberg-Marquardt method ${ }^{15}$. This method is based on steepest decent calculations and has the advantage that it can rapidly determine the parameters to minimize $\chi^{2}$. We determined that convergence is relatively sensitive to the input parameters. As a result, we used the spreadsheet program to rapidly investigate the parameter spaces to minimize $\chi^{2}$ and the our non-linear least squares program to investigate regions which gave low initial $\chi^{2}$ values. Consequently, the reader should bear in mind that the best-fit parameters reported here may not represent the global parameters which minimize $\chi^{2}$. This has some bearing on the interpretation of results and will be discussed in section 3.0.

In addition to the exponential model developed above, we also fit data using the power law formalism of equation 8 : 


$$
P=1-\exp \left(-\frac{\pi \omega_{o}^{2} C_{p}}{1-p} \int_{F_{t h}}^{F_{p k}} \frac{\left(F-F_{t h}\right)^{1-p}}{F} d F\right)
$$

Here the fitting parameters are $C_{p}, p$, and $F_{t h}$. This expression is due to O'Connell and is a variation of the Weibull formalism currently being used by Feit et al. It is discussed in detail in Appendix 1.

\subsection{Results of exponential fitting to KDP214 test data}

We applied each of these models to data obtained from KDP214. This is high quality, rapid-growth material which exhibits performance approaching that of Beamlet-era, conventionally grown KD*P. This crystal has also been extensively investigated using scattered light imaging, fluorescence imaging and photothermal mapping. In addition, it has been used in small-beam thermal and laser annealing experiments as well as tested on the Optical Sciences Laser. The 1/1 and 8/1 Scurves for OSL data were generated by integrating fluence histograms for all pinpoint densities greater than the Zeus detection threshold of $3 \mathrm{pp} / \mathrm{mm}^{3}$. Figure 3 shows the evolution of the damage probability curve as a function of laser conditioning for damage at $3 \omega$ as well as their associated best -fit curves from the exponential model. As the amount of conditioning increases, the slope of the curve (in the linear portion) decreases. The relative scarcity of data in the $8 / 1$ case is because the crystal conditioned enough so that relatively few pinpoints were generated at the fluence level of the last shot in the ramped sequence.

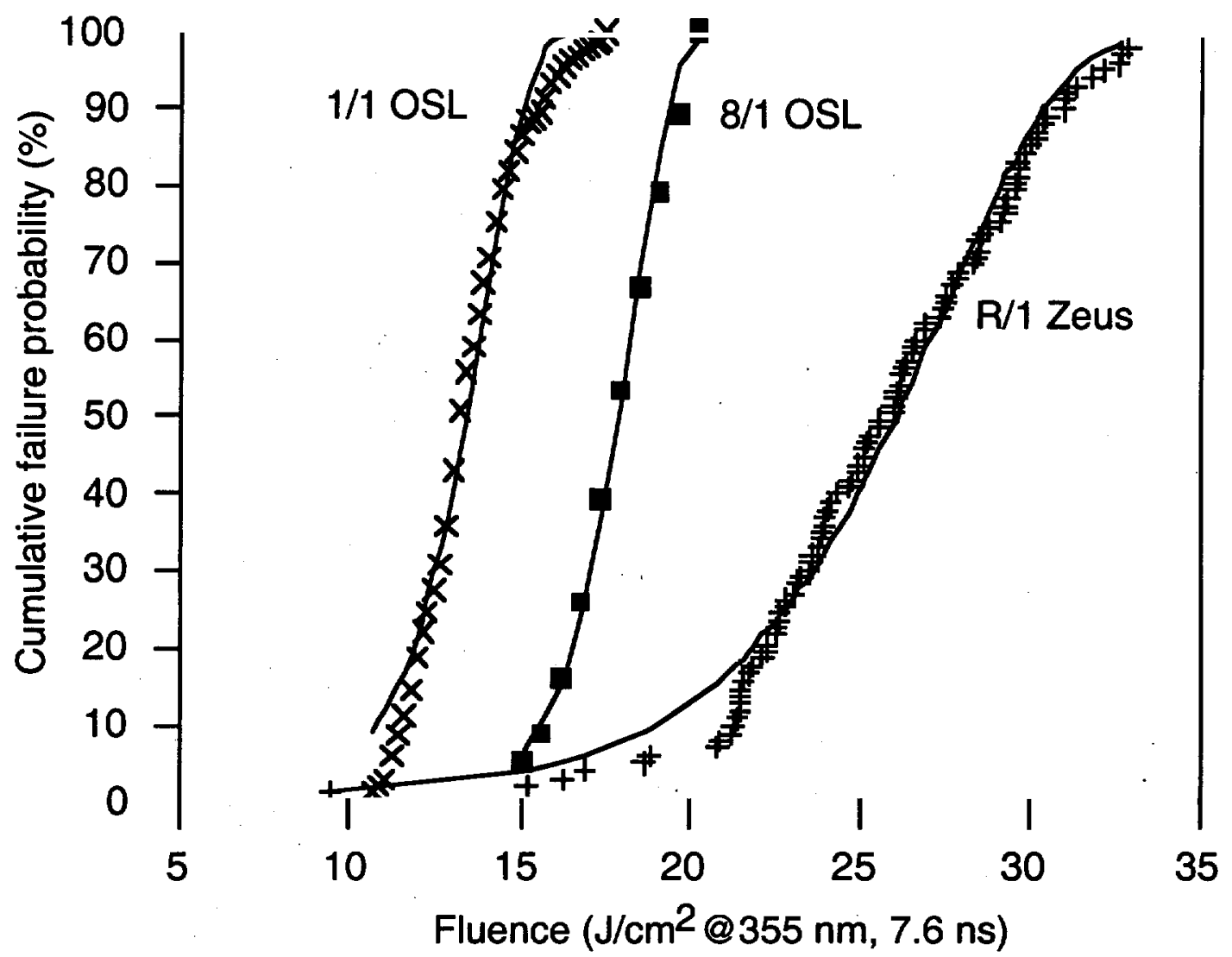

Figure 3. Best-fit curves for the exponential damage evolution model applied to KDP214 damage at $3 \omega$. The fit parameters for these curves are given in Table 1.

We start our discussion of the fit results by presenting the best fit parameters for each data curve in Table 1 as well as a plot of the data fits in Figure 3. 
Table 1. Best fit parameters for KDP214 data and exponential model fits.

\begin{tabular}{|c|c|c|c||c|c|}
\hline Test & $\mathrm{n}_{\mathrm{o}}\left(\mathrm{pp} / \mathrm{cm}^{3}\right)$ & $\mathrm{b}\left(\mathrm{cm}^{2} / \mathrm{J}\right)$ & $\mathrm{F}_{\mathrm{th}}\left(\mathrm{J} / \mathrm{cm}^{2}\right)$ & \multicolumn{2}{|c|}{ OSL parameters } \\
& & & & $\mathrm{n}_{0}\left(\mathrm{pp} / \mathrm{cm}^{3}\right)$ & $\mathrm{b}\left(\mathrm{cm}^{2} / \mathrm{J}\right)$ \\
\hline \hline $1 / 1$ OSL & $5 \times 10^{-2}$ & 0.822 & 0.01 & $7.5 \times 10^{-2}$ & 1.5 \\
\hline $8 / 1$ OSL & $5 \times 10^{-4}$ & 0.896 & 0.01 & $10^{-4}$ & 2.5 \\
\hline R/1 Zeus & 0.5 & 0.323 & 0.01 & - & - \\
\hline $1 \omega \mathrm{R} / 1$ Zeus & 1.0 & 0.098 & 0.01 & - & - \\
\hline
\end{tabular}

We chose to set the threshold fluence to $0.01 \mathrm{~J} / \mathrm{cm} 2$ thereby eliminating one fit parameter. This was done for both practical and philosophical reasons that will be discussed below. The parameters presented here were obtained by holding the value of $\mathrm{n}_{0}$ constant and varying $\mathrm{b}$ until $\chi^{2}$ was minimized. Inspection shows that the $\mathrm{n}_{0}$ values for the model probability curves have the same order of magnitude as the measured OSL parameters. The $b$ values, however, differ significantly. We shall explore the evolution of the fit curve with parameter variation and whether the results are physically meaningful in the following paragraphs and sections.

\subsection{Evolution of the exponential model with parameter variation}

In this section, we explore how the model probability curve varies with parameter variation. Figure 4 shows the damage probability curve for KDP214 at $1 \omega$ along with three model curves representing the evolution of the fit as parameters are varied.

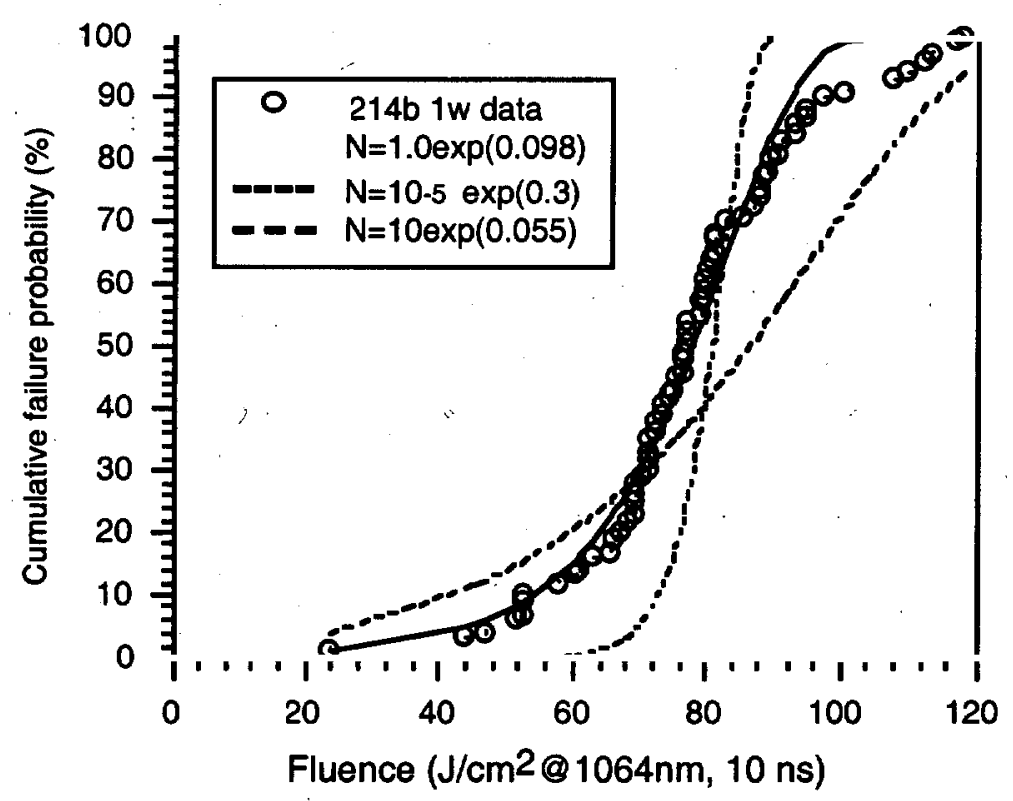

Figure 4. Damage probability data for KDP214 at $1 \omega$ and model fits representing the evolution of the curve as the parameters $n_{0}$ and $b$ are varied. $F_{t h}$ is held constant at $0.01 \mathrm{~J} / \mathrm{cm}^{2}$ for these curves.

Generally speaking, the lower $n_{0}$ is, the greater the value of $b$ must be in order to bring the fit close to the experimental data. However, these combinations also give the curve a more step-function like appearance. On the other hand, higher values of $\mathrm{n}_{0}$ require lower values of $\mathrm{b}$ to fit the data and lead to curves with lower slope. The effect of increasing the threshold fluence, $F_{t b}$, is to lower the individual damage probabilities for each fluence. Clearly, the maximum allowable threshold fluence is the lowest value in the data set and increasing $F_{\mathrm{th}}$ beyond this minimum fluence would be unphysical.

The concept of an absolute damage threshold fluence becomes somewhat nebulous in the model. While it is true that each individual test site has a well-defined damage onset, indications are that the minimum overall threshold will continue to drop 
as more sites are exposed. For example, we observed this effect for another rapid growth sample KDP347, where the 100 site, $\mathrm{R} / 1$, minimum damaging fluence was $\sim 18 \mathrm{~J} / \mathrm{cm} 2$. Ramping an additional 875 sites to fluences up to $\sim 18 \mathrm{~J} / \mathrm{cm}^{2}$ reduced the minimum damaging fluence to $5 \mathrm{~J} / \mathrm{cm}^{2}$. We expect that further exposures would eventually drive the minimum damaging fluence even lower. On the other hand, there must be a lower limit on the damaging fluence based on the damage mechanism and mechanical properties of the crystal. It appears that this threshold is low enough, particularly at $3 \omega$, so that the damage models will not be drastically affected by setting it (essentially) to zero. This does not mean, however, that the curves obtained with $F_{t h}=0.01 \mathrm{~J} / \mathrm{cm}^{2}$ are always better than those obtained for nonzero values of $F_{\mathrm{th}}$.

Also, there are numerous points in the parameter space which will give very good fits to the data, despite large differences in the parameters themselves. As an example, data for the $3 \omega, 1 / 1$ OSL damage curve is given in Table 2. This table gives the fit parameters and associated $\chi^{2}$ values. The last three entries in the table are virtually indistinguishable from each other when the data are shown on a graph. Despite the fact that very good fits to the small spot data are possible, this effect makes it very difficult to use the exponential model for predicting large-scale damage behavior. We discuss this in more detail in section 3.

Table 2. Fit parameters for KDP214, 3w, 1/1 OSL data

\begin{tabular}{|c|c|c|c|c|}
\hline $\mathrm{n}_{0}\left(\mathrm{pp} / \mathrm{cm}^{3}\right)$ & $\mathrm{b}\left(\mathrm{cm}^{2} / \mathrm{J}\right)$ & $\mathrm{F}_{\mathrm{th}}\left(\mathrm{J} / \mathrm{cm}^{2}\right)$ & $\chi^{2}$ & Comments \\
\hline \hline $10^{-1}$ & 2.0 & 0.01 & 1.524 & Best fit with b from OSL data \\
\hline $10^{-1}$ & 1.86 & 0.01 & 0.476 & Best fit using $\mathrm{n}_{0}=10^{-7}$ \\
\hline $10^{-6}$ & 1.69 & 0.01 & .388 & \\
\hline $10^{-5}$ & 1.51 & 0.01 & .303 & \\
\hline $10^{-4}$ & 1.325 & 0.01 & .216 & \\
\hline $10^{-3}$ & 1.141 & 0.01 & .1362 & \\
\hline $10^{-2}$ & .954 & 0.01 & .0813 & \\
\hline $5 \times 10^{-2}$ & .822 & 0.01 & .0800 & Best fit using $\mathrm{n}_{0}$ from OSL data \\
\hline $10^{-1}$ & .765 & 0.01 & .0972 & \\
\hline
\end{tabular}

\subsection{Results of power law fitting to KDP214 test data}

In this section, we repeat the analysis given above for the power law formalism given in equation (8). As mentioned above, this particular formalism is due to $\mathrm{O}^{\prime}$ Connell ${ }^{13}$. We give equation 8 again:

$$
P=1-\exp \left(-\frac{\pi \omega_{o}^{2} C_{p}}{1-p} \int_{F_{\text {th }}}^{F_{p k}} \frac{\left(F-F_{t h}\right)^{1-p}}{F} d F\right)
$$

The fit parameters in this model are $C_{p}, p$ and $F_{u}$. The units on $C_{p}$ are variable and depend on the value of $p$, but overall $C_{p}$ is related to the defect concentration. Inspection shows that the exponent diverges only for $p=1$, otherwise all values of $p$ are allowed. Setting $F=0$ also leads to a divergence and therefore precludes setting $F_{\mathrm{th}}=0$. Table 3 shows the best-fit parameters for KDP214 test data in analogy to the exponential case (Table 1). We only show data for the cases where $F_{t h}=0.01$, however, similar arguments regarding the role of $F_{\mathrm{th}}$ in this model apply. In addition, we show the Weibull exponent that was derived from linear least-squares fitting of the damage curve in the form of equation (3). In general, the data was obtained by holding $p$ constant and adjusting $C_{p}$ until $\chi^{2}$ was minimized.

Table 3. Best fit parameters for KDP214 data and power law model fits.

\begin{tabular}{|c|c|c|c||c|}
\hline Test & $\mathrm{C}_{\mathrm{p}}$ & $\mathrm{p}$ & $\mathrm{F}_{\mathrm{hh}}\left(\mathrm{J} / \mathrm{cm}^{2}\right)$ & Weibull exponent $(\mathrm{m})$ \\
\hline \hline 1/1 OSL & $5.1 \times 10^{-8}$ & -9.5 & 0.01 & 10.60 \\
\hline $8 / 1$ OSL & $3.9 \times 10^{16}$ & -14.4 & 0.01 & 14.90 \\
\hline R/1 Zeus & $1.98 \times 10^{-6}$ & -6.0 & 0.01 & 6.36 \\
\hline $1 \omega \mathrm{R} / 1$ Zeus & $5.25 \times 10^{-8}$ & -5.0 & 0.01 & 4.78 \\
\hline
\end{tabular}


Inspection of the data shows that $p$ values have a very wide range and the data for the $8 / 1$ case is somewhat anomalous, as was the case in the exponential model. Despite this, we see that the magnitude of $p$ is approximately equal to the Weibull exponent (i.e. $-\mathrm{p} \sim \mathrm{m})$.

\subsection{Evolution of the power law model with parameter variation}

As can be seen in Table 3, the magnitude of $C_{p}$ closely follows the value of $p$. Setting $C_{p} \sim 10^{p}$ proved to be an adequate starting point for fitting the data. Generally speaking, increasing the value of $C_{p}$ while holding the other parameters fixed increased the calculated failure probability. Decreasing $\mathrm{p}$ also increased the calculated failure probabilities and gave the curve a more step-function like appearance. In contrast, increasing the damage threshold, $\mathrm{F}_{\mathrm{th}}$, decreases the failure probability. Unlike the exponential model, this model does not allow values of $F_{t}>F_{\min }$ or a divergence will result. Figure 5 shows the evolution of the damage curve with parameter variation for the $1 \omega$ damage curve of sample KDP214.

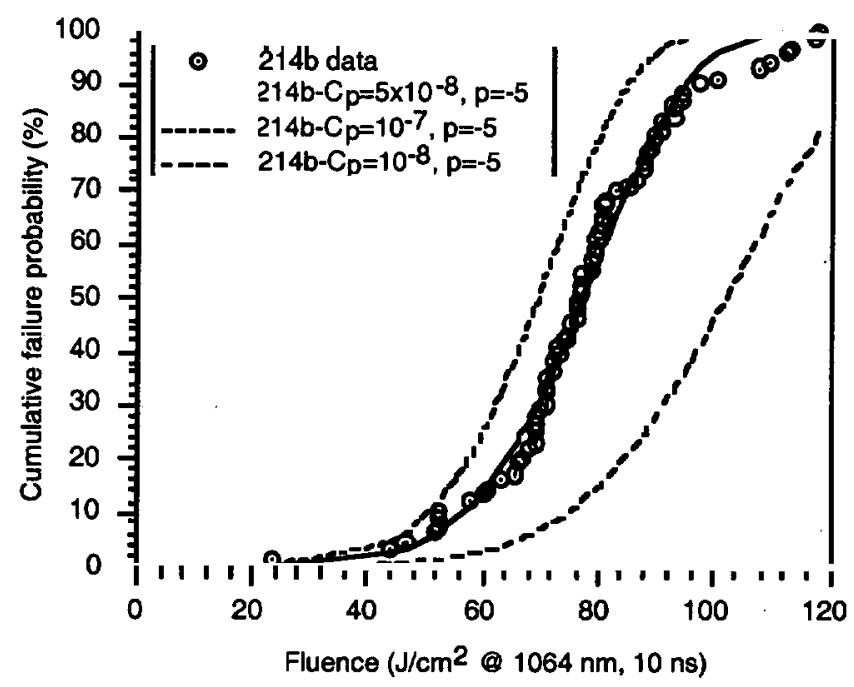

Figure 5. Power law fits to KDP214 $1 \omega$ damage data showing the evolution of curves with parameter variation.

In analogy to the exponential model, we also found that there are multiple parameter sets that give very good fits to the experimental data. We therefore encounter the same difficulty in applying these data fits to large area damage prediction.

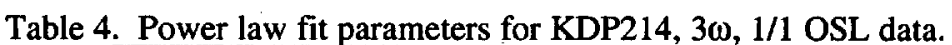

\begin{tabular}{|c|c|c|c|}
\hline $\mathrm{C}_{\mathrm{p}}$ & $\mathrm{p}$ & $\mathrm{F}_{\mathrm{th}}\left(\mathrm{J} / \mathrm{cm}^{2}\right)$ & $\chi^{2}$ \\
\hline $5.1 \times 10^{-8}$ & -9.5 & 0.01 & 0.0504 \\
\hline $1.23 \times 10^{-1}$ & -9.5 & 1.0 & 0.0565 \\
\hline $5.0 \times 10^{-1}$ & -8.5 & 0.01 & 0.0583 \\
\hline $6.25 \times 10^{-6}$ & -7.5 & .01 & 0.0908 \\
\hline
\end{tabular}

\subsection{Comparison of exponential and power law models}

Comparison of the fit data for the exponential and power law models for a number of damage curves shows that in approximately half of the cases, the power law model gives lower $\chi^{2}$ values than the exponential model. This is despite the known exponential evolution of damage in KDP from the OSL experiments. Table 5 shows the best fit $\chi^{2}$ value for each model for tests discussed in this memo.

Table 5. Comparison of exponential and power law goodness-of-fit parameter $\left(\chi^{2}\right)$. For these data there were no restrictions placed on any parameter, except that $0<\mathrm{F}_{\min }<\mathrm{F}_{\mathrm{th}}$. Consequently, some $\chi^{2}$ values may be different from those appearing in other tables. 


\begin{tabular}{|c|c|c|}
\hline Test & $\chi^{2}-$ exponential & $\chi^{2}-$ power law \\
\hline KDP214-3 $\omega, 1 / 1$ OSL & 0.0800 & 0.0504 \\
\hline KDP214-3 $\omega, 8 / 1$ OSL & 0.0136 & 0.0169 \\
\hline KDP214-3w, R/1 Zeus & 0.1133 & 0.0594 \\
\hline KDP214-1 $\omega$, R/1 Zeus & 0.1103 & 0.0794 \\
\hline DKDP586-3 $\omega, R / 1$ Zeus & 0.0454 & 0.0297 \\
\hline 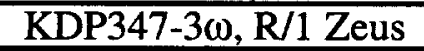 & 0.0844 & 0.1335 \\
\hline
\end{tabular}

As an example illustrating the differences between the two models, we plot the experimental data for samples KDP347 and DKDP586 (not previously discussed) along with best-fit curves for each model in Figure 6. For KDP347 both models underestimate the measured failure probability in the lower portion of the curve, with the power law being slightly worse than the exponential model. For DKDP586, which represents typical damage performance for rapid-growth DKDP, both models overestimate the failure probability in the lower portion of the curve. Here, the exponential model again fits slightly better. It is possible to attain a better fit to the lower part of the curves in both data sets, but this comes at the expense of the overall fit, with deviations now occurring in the upper portion of the curve.

\section{DISCUSSION}

The results obtained from fitting the exponential and power law models to KDP damage probability curves offer a number of paradoxes. We have seen that both models offer very precise representations of the damage curves, however, neither may be very accurate given the multiplicity of parameter sets that produce low $\chi^{2}$ values. We also see that the power law model gives better overall fits in a surprising number of cases. This is despite the fact that damage has been shown to evolve exponentially with fluence. In addition, the results from the exponential model do not agree well with measured damage evolution parameters for the OSL tests.

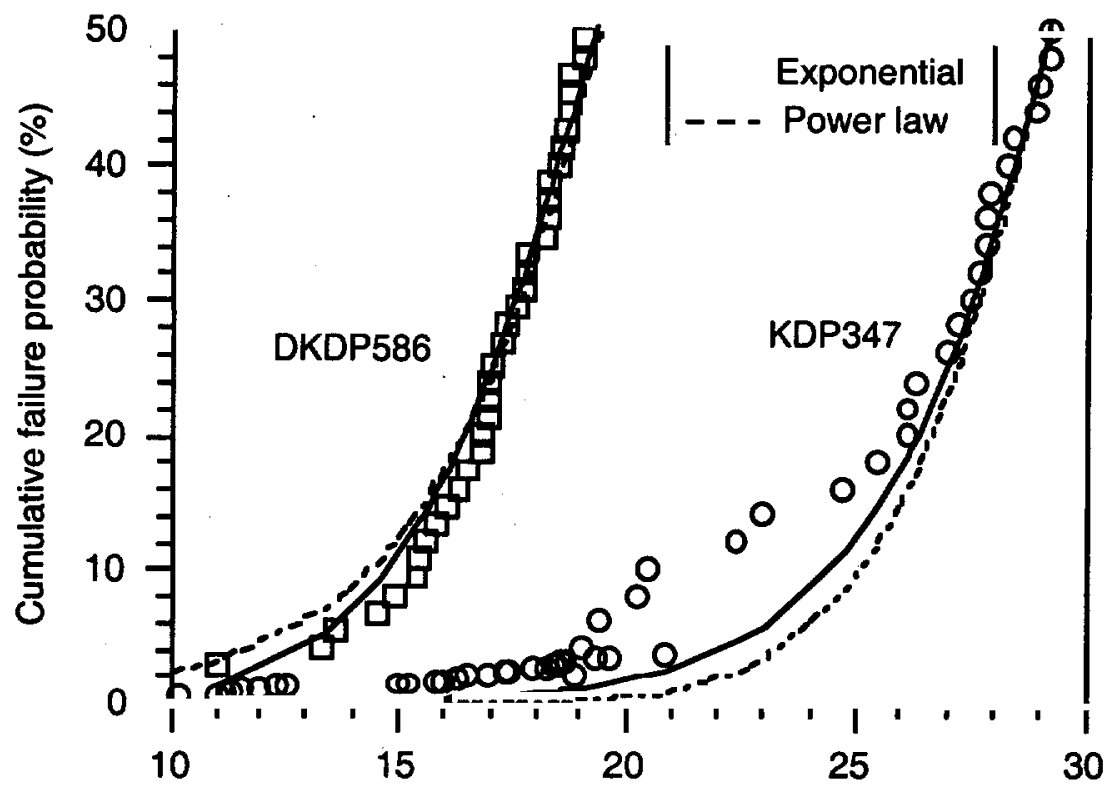

Fluence $\left(\mathrm{J} / \mathrm{cm}^{2} @ 355 \mathrm{~nm}, 7.6 \mathrm{~ns}\right)$

Figure 6. Comparison of exponential and power law models for KDP347 and DKDP586, 3w damage data. Neither model fits the lower portion of the either data set curve well with optimal overall fit parameters. The $\chi^{2}$ values for each model are listed in Table 5 .

There are a number of possible explanations for these observations and some general comments are in order. First, we should not hold too strongly to the notion that any of the damage curves shown are reproducible in more than an average sense. 
Previous work ${ }^{16}$ has shown that repeated damage testing of the same sample yields different, but similar curves for each test, from which a cumulative curve may be constructed. This is to be expected based on limited sampling of the underlying damage distribution. In addition, there is always a fluence measurement error, which is implicit in every damage curve. For the Zeus system, the $1 \omega$ and $3 \omega$ fluence errors are $\pm 5 \%$ and $\pm 15 \%$ respectively. Combining the sampling and fluence measurement uncertainties gives a typical error in the damage curve of approximately $\pm 20 \%$ for a 100 site, $3 \omega \mathrm{R} / 1$ damage test on KDP. We have not explored the effect of these errors on fit parameters in this report.

We also do not take into account fluctuations in the damage density in determining the damage evolution equation. The pinpoint density can vary by two orders of magnitude for a given fluence in large beam N/1 tests, as was shown in Figure 2 . This behavior is also observed in small beam tests where damage may be characterized by a single, or many pinpoints. It is therefore only possible to determine the damage evolution equation in an average sense. It should not be entirely surprising that the exponential parameters measured for OSL test samples are different from those obtained for the exponential model, given the experimental errors involved. It is encouraging that the $n_{0}$ values can be made to agree reasonably well, but the large discrepancy in the $b$ values is disturbing (Table 1).

The similarity of fits achievable between the models is to be expected because both the exponential and power law functions are powerful functions, mathematically speaking. Also, three-parameter equations offer wide latitude in the choice of parameters to achieve a fit. Even reducing the equations to two fitting parameters by setting $F_{t h}=0$ doesn't appear to restrict the degeneracy of solutions possible. The spreadsheet calculations indicate that the topology of the $\chi^{2}\left(n_{0}, b, F_{b b}\right)$ or $\chi^{2}\left(C_{p}, p\right.$, $F_{t h}$ ) space is filled with "holes" which are approximately the same depth. But, the spreadsheet approach doesn't allow investigation of the parameter spaces with enough resolution to determine whether a global minimum for $\chi^{2}$ exists. Indeed, the Levenberg-Marquardt program mentioned earlier will offer us the ability to map the parameter spaces at much higher resolution. Once this program is fully implemented, it should be possible to determine whether a single, global, best fit exists.

The work presented above represents an extension of existing theory to bulk damage in KDP. At this stage it is at best difficult to obtain meaningful results using these models to predict large area damage behavior. The exponential model offers direct calculation of parameters that have been, or can be measured, however the agreement between fit and measured parameters is generally not good. On the other hand, the power law model returns values of $p$ which are close to the Weibull exponent. The disadvantage in this model is extracting the defect concentration from $\mathrm{C}_{\mathrm{p}}$, which has units that depend on $\mathrm{p}$.

Despite the difficulties we discovered in attempting to derive large area damage behavior from small area tests, it is also clear that a model for laser conditioning of KDP needs to be developed. The slopes of the damage curves are consistently lower for $\mathrm{R} / 1$ tests than for $\mathrm{S} / 1$ tests. The $\mathrm{R} / 1$ test represents the maximum level of conditioning attainable at each test site while the $\mathrm{N} / 1$ exposures in OSL or Slab Lab test environments would offer less laser conditioning. Even if a better fitting approach is found, a conditioning model would need to be incorporated in it to prevent underestimating damage when fitting $R / 1$ data to predict damage in large area N/1 beam environments. In order to develop a conditioning model, we need to go beyond the current phenomological observations and make some assumptions about the nature of the damage mechanism and the conditioning process. Work on understanding the damage mechanism is well underway and indicates that nanometer scale absorbers are the likely source, however nothing currently exists regarding a laser conditioning model which correlates well with this hypothesis.

We would like to thank Mike Feit, Alexander Rubenchik , Jim DeYoreo and Alan Burnham for helpful discussions and critical reading of the text.

Work performed under the auspices of the United States Department of Energy by Lawrence Livermore National Laboratory under Contract No. W-7405-ENG-48.

\section{REFERENCES}

1) M. Runkel , M Yan, J. DeYoreo, N. Zaitseva, "The effect of impurities and stress on the damage distributions of rapidly grown KDP crystals," SPIE Proceedings, Volume 3244, 211-222, 1997

2) M. Runkel, W. Williams, J. DeYoreo, "Predicting NIF THG performance," SPIE Proceedings, Volume 3578, 322-334, 1998 
3) M. Feit, A. Rubenchik, M. Kozlowski, F. Genin, S. Schwartz, L. Sheehan, "Extrapolation of damage test data to predict performance of large-area NIF optics at $355 \mathrm{~nm}$," SPIE Proceedings, Volume 3578, 226-231, 1998

4) W. Weibull, "A statistical distribution function of wide applicability," J. Appl. Mech, 18, 293-297 (1951)

5) M. Feit, "Analysis of large area damage test data for HDT optics,"3 3 damage mechanism-meeting notes, May 26, 1998

6) F.V. Bunkin, V.V. Savranskii, "Optical breakdown of gases induced by the thermal explosion of suspended macroscopic particles," Sov. Phys. JETP, 38(6), 1091-1097, (1975)

7) A.V. Besserab, S.B, Kormer, D.V. Pavlov, A.I. Funtikov, "Statistical relationship governing the surface damage of optical glass by wide laser radiation beams," Sov. J. Quantum Electron. 7(2), 181-185 (1977)

8) S.R. Foltyn, "Spotsize effects in laser damage testing," NBS special pub 368-379 (1981)

9) J.O. Porteus, S.C. Seitel, “Absolute onset of optical surface damage using distributed defect ensembles," Applied Optics, 23(21), 3796-3805, (1984)

10) J.O. Porteus, J.B. Franck, S.C. Seitel, S.D. Allen, "Defect characteristics of optical surfaces using pulsed laser damage methods," Optical Engineering, 25(10), 1171-1176 (1986)

11) B.G. Gorshkov, Y.K. Danileiko, A.A. Manenkov, A.M. Prokhorov, A.V. Sidorin, "Size effect and statistics of laser damage to alkali-halide crystals at the $10.6 \mu \mathrm{m}$ wavelength," Sov. J. Quantum Electron. 11(1), 81-84 (1981)

12) Y.K. Danileiko, Y.P. Minaev, B.N. Nikolaev, A.V. Sidorin, "Determination of the characteristics of microdefects from statistical relationships governing laser damage to solid transparent materials," Sov. J. Quantum Electron. 11(11), 14451449 (1981)

13) R.M. O'Connell, "Onset threshold analysis of defect-driven surface and bulk laser damage," Applied Optics, 31(21), 4143-4153 (1992)

14) M. Runkel, J. DeYoreo, W. Sell, D. Milam, "Laser conditioning study of KDP on the Optical Sciences Laser," SPIE Proceedings, Volume 3244, 51-63, 1997

15) "Numerical Recipes in C, The Art of Scientific Computing," W. H. Press et al., Cambridge University Press, pp. 688, 1992

16) M. Runkel, NIF-6730, "Monte Carlo simulation of the R/1 automated damage test," SPIE Proceedings, Volume 3578, 277-287, 1998

17) P. Duxbury, P. Leath, P. Beal, "Breakdown properties of quenched random system: The random-fuse network," Physical Review B, 36(1), 367-380, 1987 